\title{
Generating Politically-Relevant Event Data
}

\author{
John Beieler \\ Human Language Technology Center of Excellence \\ Johns Hopkins University \\ jbeieler1@jhu.edu
}

\begin{abstract}
Automatically generated political event data is an important part of the social science data ecosystem. The approaches for generating this data, though, have remained largely the same for two decades. During this time, the field of computational linguistics has progressed tremendously. This paper presents an overview of political event data, including methods and ontologies, and a set of experiments to determine the applicability of deep neural networks to the extraction of political events from news text.
\end{abstract}

\section{Introduction}

Automated coding of political event data, or the record of who-did-what-to-whom within the context of political actions, has existed for roughly two decades. This type of data can prove highly useful for many types of studies. Since this data is inherently atomic, each observation is a record of a single event between a source and a target, often at the daily level, it provides a disaggregated view of political events. This means that the data enables the examination of interactions below the usual monthly or yearly levels of aggregation. This approach can be used in a manner consistent with traditional hypothesis testing that is the norm within political science (Cederman and Gleditsch, 2009; Gleditsch, 2012; Goldstein et al., 2001). Additionally, event data has proven useful in forecasting models of conflict since the finer time resolution allows analysts to gain better leverage over the prediction problem than is possible when using more highly aggregated data (Arva et al., 2013; Brandt et al., 2014; Brandt et al., 2013; Brandt et al., 2011).

The methods used to generate this data have remained largely the unchanged during the past two decades, namely using parser-based methods with text dictionaries to resolve candidate noun and verb phrases to actor and event categories. The underlying coding technologies have moved slowly in updating to reflect changes in natural language processing (NLP) technology. These NLP technologies have now advanced to such a level, and with accompanying open-source software implementations, that their inclusion in the event-data coding process comes as an obvious advancement. Given this, this paper presents the beginnings of how modern natural language processing approaches, such as deep neural networks, can work within the context of automatically generating political event data. The goal is to present a new take on generating political event data, moving from parser-based methods to classifier-based models for the identification and extraction of events. Additionally, this paper serves as an introduction to politically-relevant coding ontologies that offer a new application domain for natural language processing researchers.

\section{Political Event Ontologies}

Political event data has existed in various forms since the 1970s. Two of the original political event datasets were the World Event Interaction Survey (WEIS) and the Conflict and Peace Data Bank (COPDAB) (Azar, 1980; McClelland, 1976). These two datasets were eventually replaced by the projects created by Philip Schrodt and various col- 
laborators. In general, these projects were marked by the use of the Conflict and Mediation Event Observations (CAMEO) coding ontology and automated, machine-coding rather than human coding (Gerner et al., 2001; Schrodt et al., 2008). The CAMEO ontology is made up of 20 "top-level" categories that encompass actions such as "Make Statement" or "Protest." Each of these twenty top-level categories contains finer-grained categories in a hierarchical manner. For example, the code 14 is the top-level code for "Protest" with the sub-code 142 representing a general demonstration or rally. Under the code 141, "Demonstrate or rally," is code 1411 which codes "demonstrate or rally for leadership change." Thus, as one moves down the hierarchy of CAMEO, more fine-grained events are encountered. All told, this hierarchical scheme contains over 200 total event classifications. This ontology has served as the basis for most of the modern event datasets such as the Integrated Crisis Early Warning System (ICEWS) (O'Brien, 2010), the Global Database of Events, Language, and Tone (GDELT) ${ }^{1}$, and the Phoenix ${ }^{2}$ dataset.

The defining feature of the CAMEO ontology is the presence of a well-defined ontology consisting of verb phrases and noun phrases used in the coding of actions and actors. For each of the 200+ categories of event types in CAMEO, there exists a list of verb phrases that define a given category. Similarly, the scope of what is considered a valid actor within CAMEO is defined by the noun phrases contained in the actor dictionaries. Thus, CAMEO is scoped entirely by the human-defined noun and verb phrases contained within underlying text dictionaries. The creation of these dictionaries is a massively costly task in terms of human labor; to date each phrase in the dictionaries was identified, defined, and formatted for inclusion by a human coder.

\subsection{Lower Resolution Ontologies}

While the CAMEO ontology offers fine-grained coding of political events within the 20 top-level categories, a small but convincing set of literature suggests that this level of granularity is not necessary to answer many of the questions to which event data is

\footnotetext{
${ }^{1}$ gdeltproject.org

${ }^{2}$ phoenixdata.org
}

applied. Schrodt (2006), for example, suggests that dividing the CAMEO ontology into much lowerresolution categories, known as QuadClasses, provides enough information to perform accurate out-of-sample forecasts of relevant political events. Additionally, Schein et al. (2016) indicates that it is possible to recover this latent structure from coded events. These QuadClass variables, which are divided along conflict/cooperation and material/verbal axises, capture the information described in the above papers. As seen in Figure 1, a given event can be placed somewhere within the resultant quadrants based on what valence the event has (conflict or cooperation) and what realm the event occurred (verbal or material).

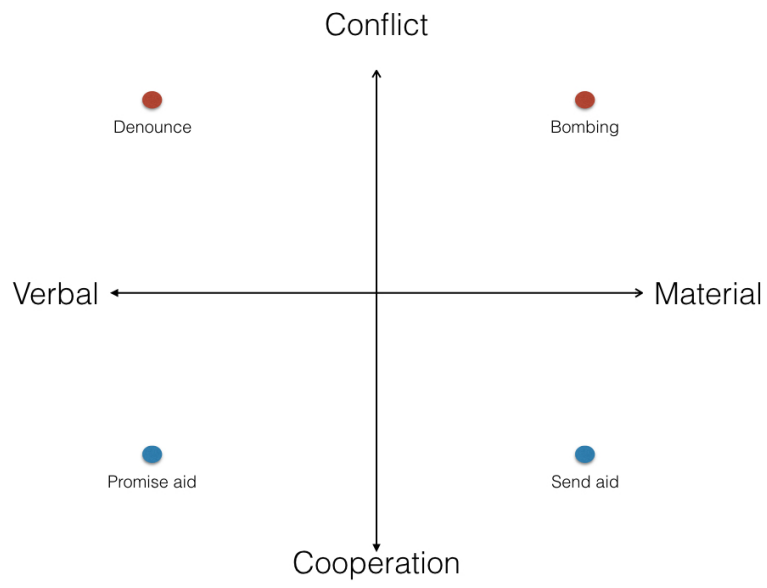

Figure 1: QuadClass Event Quadrants

Since these QuadClass variables capture much of the information necessary, the methods discussed within this paper focus on this rather than the full CAMEO coding ontology.

While the QuadClass variables lose some of the benefits of the more disaggregated, 200+ code CAMEO ontology, such as those outlined in Cederman and Gleditsch (2009), the usage of these lower-resolution categories is acceptable for a few reasons. First, as mentioned above, these categories capture a significant portion of the useful variance in the usual dependent variables of interest within political science. Second, these lower-resolution categories help resolve much of the ambiguity in the 
more detailed CAMEO categories. For example, the "Protest" category of CAMEO encompasses events such as demonstrations as well as political leaders verbally protesting the actions of another entity. Because of this, what seems to be a benefit of CAMEO, fine-grained differentiation between categories, becomes a possible point of confusion. Finally, and most importantly, the broad goal of the approach outlined in this paper is to enable researchers to develop application-specific ontologies that allow for categories that address the needs of a particular research question rather than attempting to make use of an ontology imperfectly suited to the task at hand. Thus, the hope is that more ontologies will proliferate to better support a researcher's specific needs.

\section{Current Approaches}

The current state-of-the-art for CAMEO-coded political event extraction is presented by the PETRARCH $2^{3}$ coder. $^{4}$ The main features of PETRARCH2 include a deep reliance on information from a constituency parse tree. The default parse comes from the Stanford CoreNLP software (Manning et al., 2014). The exact operational details of PETRARCH2 are beyond the scope of this paper, with a complete explanation of the algorithm available in Norris (2016); it should suffice to say that this second version of PETRARCH makes extensive use of the actual structure of the parse tree to determine source-action-target event codings. This change to be more tightly coupled to the tree structure of the sentence, as compared to previous coders such as TABARI (Schrodt, 2001), allows for a clearer identification of actors and the assignment of role codes to the actors, and a more accurate identification of the who and whom portions of the who-did-whatto-whom equation.

At its heart, PETRARCH2 is still software to perform a lookup of terms in a set of text dictionaries. Given this, if the terms identified by the program are incorrect then the final event coding will also be incorrect. Additionally, if the terms identified by PETRARCH2 are not in the dictionaries, but would still be associated with a valid actor or event coding, then

\footnotetext{
${ }^{3}$ https://github.com/openeventdata/petrarch2

${ }^{4}$ Other coders exist, such as BBN's ACCENT coder, but is not currently publicly available. PETRARCH2 and ACCENT approach event coding in roughly the same manner, however.
}

no event is coded. This means that no matter the algorithmic design of the event coder, the output will remain constrained by the verb and actor dictionaries.

The primary issue with these methods is twofold. First, the parser-based methods rely on humancreated dictionaries. As noted above, this is a labor intensive task that is not easily replicable for expansion into new ontologies; CAMEO has become the de-facto coding standard for political events largely owing to the existence of the text dictionaries created over the course of two decades. O'Connor et al. (2013) introduced a method that potentially solves the issue of developing verb patterns for the coding of political events. This method still does not address many of the other issues present with the current approaches to generating political event data, such as a reliance on syntactic parsers. The second issue, owing to the reliance on text dictionaries and parsers for the extraction of events, is the exclusively English-language nature of all available event datasets. The next section introduces an alternative to these parser-based methods that is applicable across ontologies, is tune-able for a given problem set, and is capable of working cross-lingually.

\section{Statistical Approaches}

In order to replace the parser-based methods for identifying an event, a new system must indentify to which of the four QuadClass variables, Material Conflict, Material Cooperation, Verbal Conflict, or Verbal Cooperation, the event belongs. To accomplish this, this paper makes use of convolutional neural nets.

This paper considers two neural architectures to classify a political event. The first is a 1-dimensional ConvNet with pre-trained word embedding features as described in Kim (2014). In short, it is a relatively shallow network with three parallel convolutional layers and two fully-connected layers. The fully-connected layers contain 150 and 4 units each. Dropout is applied to the two fully-connected layers so meaningful connections are learned by the model. The details of the model layers are in Table 1.

The second model is a character ConvNet based on the work by Zhang et al. (2015). The character ConvNet is a modified implementation since mul- 


\begin{tabular}{cccc}
\hline Layer & Frame & Kernel & Pool \\
\hline 1 & 256 & 3 & 2 \\
2 & 256 & 4 & 2 \\
3 & 256 & 5 & 2 \\
\hline
\end{tabular}

Table 1: Word-based convolutional layers.

\begin{tabular}{cccc}
\hline Layer & Frame & Kernel & Pool \\
\hline 1 & 256 & 7 & 3 \\
2 & 256 & 3 & N/A \\
3 & 256 & 3 & N/A \\
4 & 256 & 3 & 3 \\
\hline
\end{tabular}

Table 2: Character-based convolutional layers.

tiple experiments determined the full specification in Zhang et al. (2015) underperformed other specifications. The architecture for the character ConvNet consists of 3 convolutional layers and 3 fullyconnected layers. The convolution layers are detailed in Table 2. The fully connected layers have 1024,1024 , and 4 output units, respectively.

\section{Data}

The datasets used in this paper are shown in Table 3. Each of the "soft-labelled" datasets has QuadClass labels applied to them by PETRARCH2. The use of PETRARCH2 is necessary in order to generate enough training samples for the various classification algorithms to gain leverage over the classification task. The English corpus consists of data scraped from various online news media sites. The Arabic corpus labels are obtained via the use of a sentence-aligned English/Arabic corpus. ${ }^{5}$ Thus, if a sentence is labelled as Material Conflict in the English corpus, that label is transferred to the aligned sentence in the Arabic corpus. If multiple alignments occur the label is transferred to each of the relevant sentences. The next dataset is the same set of labelled Arabic sentences that were run through the machine-translation software Joshua (Weese et al., 2011). These datasets provide information for three experiments: English-language, Arabic-language, and machine translated English.

\footnotetext{
${ }^{5}$ The specific corpus is available at https://www.ldc.upenn.edu/collaborations/pastprojects/gale/data/gale-pubs.
}

\begin{tabular}{lc}
\hline Dataset & Sentences \\
\hline Soft-labelled English & 49,296 \\
Soft-labelled Arabic & 11,466 \\
Machine-translated Arabic & 3,931 \\
\hline
\end{tabular}

Table 3: Data source type and size

\begin{tabular}{lc}
\hline \hline Model & Accuracy \\
\hline \multicolumn{2}{c}{ Word-based models } \\
\hline English Input & 0.85 \\
Native Arabic Input & 0.25 \\
Machine-translated Input & 0.60 \\
\hline \multicolumn{2}{c}{ Character-based models } \\
\hline English input & 0.94 \\
Arabic input & 0.93 \\
\hline \hline
\end{tabular}

Table 4: Accuracy scores for Category Classifier

\section{Experiments}

Table 4 shows the results of various models for classifying a sentence into one of four QuadClasses. Across the board, it is clear that the character-based ConvNets perform much better than the word-based models. The difference is less drastic for Englishlanguage inputs, a $9 \%$ difference in accuracy. For Arabic-language inputs, however, the difference is striking. The character model is over $20 \%$ more accurate than the word-based model. This is likely due to issues with tokenization and morphology when dealing with the word-based models. Even more impressive is the ability of the Arabic-language models to perform well even with a relatively small corpus of 11,466 coded sentences. These results demonstrate that character-based ConvNets are appropriate and powerful models for the classification of politically-relevant events.

\section{Conclusion}

This paper has demonstrated that modern approaches to natural language processing, specifically deep neural networks, offer a promising avenue for the extraction of politically-relevant events. The methods shown in this paper can work across both ontologies and languages offering a level of flexibility unseen in the realm of CAMEO-coded political event data. The implementation of these methods will allow for the exploration of languages and on- 
tologies, as an example expanding beyond the limits of CAMEO to code events such as crime events in Spanish-language news sources, that will open new avenues of social science research.

While these methods are promising, there is still much work left to develop a fully operational event extraction pipeline. In terms of classifying events, there is still the issue of handling the many nuances of event coding. For example, if a meeting occurs between three actors that would typically lead to nine coded events when handling the various combinations of actors and binary relations. Additionally, the methods presented on this paper do not touch upon the extraction of actor information. This is another area for which modern NLP approaches, such as semantic role labelling, are highly applicable and will likely improve on the state-of-the-art.

\section{Acknowledgments}

This work was supported by the DARPA Quantitative Crisis Response program.

\section{References}

Bryan Arva, John Beieler, Ben Fisher, Gustavo Lara, Philip A. Schrodt, Wonjun Song, Marsha Sowell, and Sam Stehle. 2013. Improving forecasts of international events of interest. Paper presented at the European Political Studies Association meetings, Barcelona, June 2013.

Edward E. Azar. 1980. The conflict and peace data bank (copdab) project. Journal of Conflict Resolution, 24:143-152.

Patrick T Brandt, John R Freeman, and Philip A Schrodt. 2011. Real time, time series forecasting of inter-and intra-state political conflict. Conflict Management and Peace Science, 28(1):41-64.

Patrick T Brandt, John R Freeman, Tse min Lin, and Philip A Schrodt. 2013. Forecasting conflict in the cross-straits: Long term and short term predictions. Annual Meeting of the American Political Science Association.

Patrick T Brandt, John R Freeman, and Philip A Schrodt. 2014. Evaluating forecasts of political conflict dynamics. International Journal of Forecasting, 30(4):944962.

Lars-Erik Cederman and Kristian Skrede Gleditsch. 2009. Introduction to special issue of "disaggregating civil war". Journal of Conflict Resolution, 24(4):590617.
Deborah J. Gerner, Philip A. Schrodt, Omur Yilmaz, and Rajaa Abu-Jabr. 2001. Conflict and mediation event observations (cameo): A new event data framework for the analysis of foreign policy interactions. American Political Science Association, Boston, August 2002.

Nils Petter Gleditsch. 2012. Whither the weather? climate change and conflict: Introduction to whither the weather? climate change and conflict: Introduction to special issue. Journal of Peace Research, 49(1):3-9.

Joshua S. Goldstein, Jon C. Pevehouse, Deborah J. Gerner, and Shibley Telhami. 2001. Dynamics of middle east conflict and u.s. influence, 1979-97. Journal of Conflict Resolution, 45(5):594-620.

Yoon Kim. 2014. Convolutional neural networks for sentence classification. In Proceedings of the 2014 Conference on Empirical Methods in Natural Language Processing (EMNLP), , pages 1746-1751, October.

Christopher D. Manning, Mihai Surdeanu, John Bauer, Jenny Finkel, Steven J. Bethard, and David McClosky. 2014. The Stanford CoreNLP natural language processing toolkit. In Proceedings of 52nd Annual Meeting of the Association for Computational Linguistics: System Demonstrations, pages 55-60.

Charles A. McClelland. 1976. World event/interaction survey codebook.

Sean P. O'Brien. 2010. Crisis early warning and decision support: Contemporary approaches and thoughts on future research. International Studies Review, 12(1):87-104.

Brendan O'Connor, Brandon Stewart, and Noah A. Smith. 2013. Learning to extract international relations from political context. In Proceedings of the Annual Meeting of the Association for Computational Linguistics (ACL), pages 1094-1104.

Aaron Schein, Mingyuan Zhou, David M. Blei, and Hanna Wallach. 2016. Bayesian poisson tucker decomposition for learning the structure of international relations. In Proceedings of the 33rd International Conference on Machine Learning, New York, NY, USA.

Philip A. Schrodt, Omur Yilmaz, Deborah J. Gerner, and Dennis Hermrick. 2008. Coding sub-state actors using the cameo (conflict and mediation event observations) actor coding framework. In International Studies Association, San Francisco, March 2008.

Philip A. Schrodt. 2001. Automated coding of international event data using sparse parsing techniques. Paper presented at the International Studies Association, Chicago, 21-24 February 2001.

Philip A Schrodt. 2006. Forecasting conflict in the balkans using hidden markov models. In Robert Trappl, editor, Programming for Peace: ComputerAided Methods for International Conflict Resolution 
and Prevention, pages 161-184. Kluwer Academic Publishers, Dordrecht, Netherlands.

Jonathan Weese, Juri Ganitkevitch, Chris CallisonBurch, Matt Post, and Adam Lopez. 2011. Joshua 3.0: Syntax-based machine translationjoshua 3.0: Syntaxbased machine translation joshua 3.0: Syntax-based machine translation with the thrax grammar extractor. In Proceedings of the 6th Workshop on Statistical Machine Translation, pages 478-484, Edinburgh, Scotland, UK, July.

Xiang Zhang, Junbo Zhao, and Yann LeCun. 2015. Character-level convolutional networks for text classification. In Advances in Neural Information Processing Systems 28 (NIPS 2015). 